\title{
Information Presentation: considering on-line User Confidence for effective engagement
}

\author{
Elahe Kani-Zabihi, Lizzie Coles-Kemp, Martin Helmhout
}

\begin{abstract}
In order to design on-line services that are able to support the end-user in making informed choices about when and how to disclose personal information, a close understanding of the relationship between privacy and confidence is therefore needed. UK citizens accessing on-line services have privacy concerns about sharing personal information with government organizations. The physical distance between service user and service provider (increased by on-line service delivery) can reduce confidence in the management of personal information. A close understanding of the relationship between user confidence and information presentation can suggest new design principles to support them in making informed choices about when and how to disclose personal information. This paper presents the result of three user studies to understand user confidence with relation to graphical information presentation, which led to three distinct types of confidence: Institutional; Technological; and Relationship. The final study represents the impact of using graphical information presentation on users' privacy concern and their confidence in using on-line services. The result indicated service users' privacy concerns decrease when their privacy awareness increase.
\end{abstract}

\section{Introduction}

The work presented in this paper is part of a project (2009-2012) entitled Visualisation and Other Methods of Expression (VOME) whose main objective was to develop methods of expressing privacy that enable a wider range of privacy concerns to be articulated and offer a broader variety of privacy protection responses [1, 2, 4, 5]. This project was part of a wider movement $[3,6,9,11]$ focused on gaining a greater understanding of information practices. This paper represents the impact of using graphical information presentation on users' privacy concern and their confidence in using on-line services.

\subsection{Trust and Confidence}

In an attempt to learn more about confidence in current literature, we learned confidence is considered to be an aspect of trust. It has long been understood that trust is

adfa, p. 1, 2011.

(C) Springer-Verlag Berlin Heidelberg 2011 
socially-constructed and multi-faceted. It is also understood that trust in an on-line service covers many different aspects: Trust in technology and Trust in the institution that delivers the service. Technological trust represents trust by individuals or institutions in technologies, from a reliability and security perspective. When users believe that the system will protect their safety and security then there is a technological trust in that system. Trust can be dependent on: perceptions of technology; trust in the security mechanisms used to secure transactions [7] and confidence in the reliability of the whole system [10]. For example, e-services with restricted access have gained users' confidence [6]. Furthermore, achieving desirable user experience goals is possible by being more responsiveness in communications. In order to build trust, service providers should work on their relationships with service users. Kuriyan et. al. [7] reports two types of trust which define relationships: Relational trust and Process-based trust. Relational trust is where trust is being a property of relations between different social actors both institutions and individuals. When there is a good relationship between a user and other actors (other users, service provider and third parties) there is a relational trust between them. Process-based trust involves an incremental "give and take" process. The institutional trust (or confidence) is built inductively through experiences and reputation. Smith [10] reported users' positive experience during their interaction with the e-service had influenced their trust on the institution. The less confidence users say they have the less likely they are to carry out a range of activities and transactions online. This has ramifications for on-line engagement and the successful delivery of public services on-line. Creating conditions in which end-users can build up meaningful confidence in a service provider's ability to manage their personal data is important. Quality of data also potentially increases the effectiveness of the services that can be offered. To define confidence, we use Luhmann's [8] definition: "confidence takes place where actions are executed under the assumption expectations will be met”. This definition is used because it most accurately reflects the service user attitude under which personal information is disclosed. 


\section{Background}

Our first user study included interviews of 56 participants with varying levels of ICT literacy. The purpose of this study was to observe users' privacy practices and hear their on-line privacy concerns during their interaction with the on-line registration process. The main objective was to gather users' expectations from an on-line service provider. The result obtained from the study has been published [5]. We learned certain level of confidence in management of personal information should exist before one discloses accurate personal data. Participants showed a desire to have a service design that provided spaces and tools through which they could think through personal information disclosure questions. Service users can have low confidence in a service provider's ability to protect their personal information even if those service users trust the overall brand. Wretchedly, contemporary on-line service designs have failed to support users to build their confidence. It was discovered users with privacy concern adopted one of the following strategies: Give false information; Discontinue with registration; Continue with registration, give accurate information but reduce the degree of on-going service engagement. However, the initial study only gave a partial picture as to the dimensions of confidence that affect a citizen's feelings about personal information disclosure in on-line contexts. Further studies were set up to develop a more complete picture.

\section{The digital intervention}

In order to better understand the dimensions of confidence and their relevance to the management of personal information we developed a digital intervention. This was of an on-line registration process which represented a mock-up council, named Your Local Council (YLC). YLC (Figure 1) offered an on-line smartcard registration service for citizens to use for local public services which adopted the smartcard for delivering the following services: a Library service, a Local Shops discount scheme and Local Transport. The prototype was designed according to a combination of user require- 
ments, HCI and CRM principles obtained from the first user study [5]. We asked participants to engage with YLC website by assuming the role of a citizen and imagine the council to be their 'real' council.

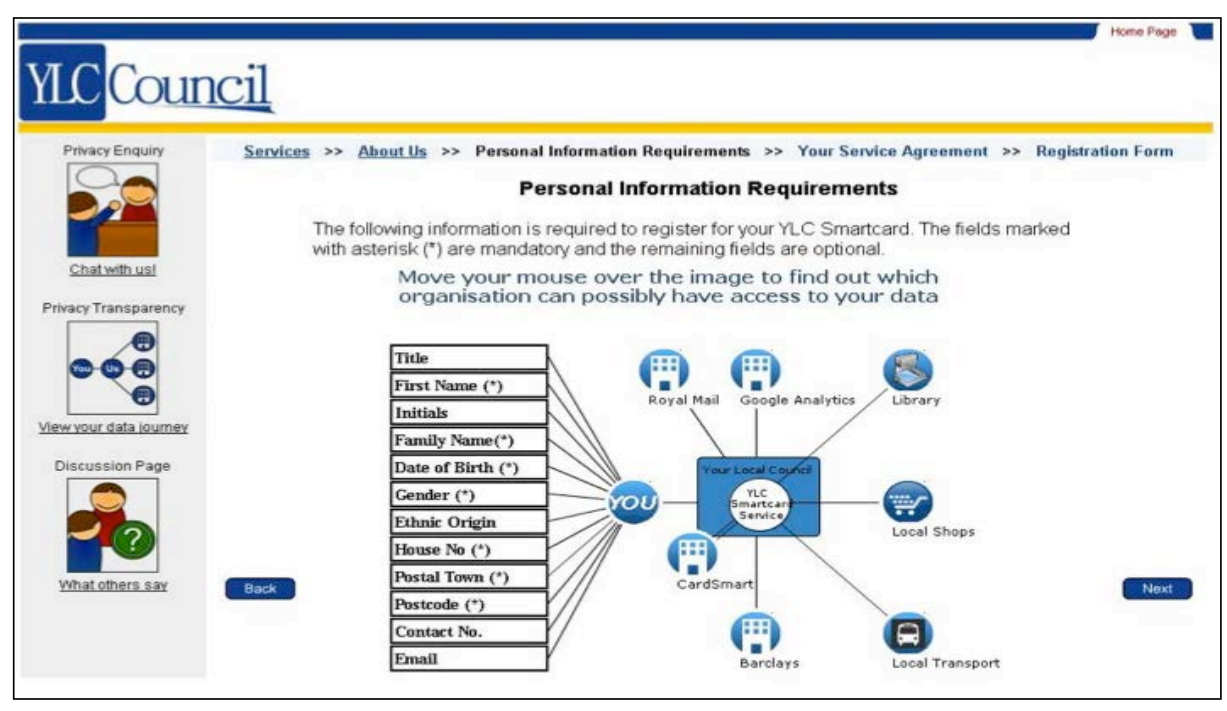

Fig. 1. YLC $-1^{\text {st }}$ Prototype

\subsection{The first prototype}

In the second user study, we were interested to know users' opinion of our new graphical information presentation design, which was an interactive data flow map (Figure 2) named Social Translucence Map (ST map). The map showed what data, in this fictional context, would be needed when eventually the user registers for a particular service and who (local service providers) will have access to what part of their personal information. Users could hover the cursor over the map to receive further information. After the introduction to smartcard services provided by YLC, users were asked to interact with the website which guides them through a sequential registration process. Every page has the same layout and contains three icons on the left side which provides three probes (left hand panel in Figure 1). Each probe enables a different kind of communication: interaction with the service provider (privacy enquiry), interaction with other service users (discussion page) and visual representations of information flows to third parties (ST map). The probes are designed to explore how different technological 
approaches can help to build awareness of the privacy features of the registration process. These probes have been fully described in [5]. The ST map is one of the Privacy Transparency probe which was used by researchers to explore the effect of transparency on both the confidence in a service provider's ability to protect personal information and on the effectiveness of this approach to increase privacy awareness.

In order to explore the different dimensions to confidence and their relationship with personal information disclosure, a mixed methods approach was used: Questionnaire; Engagement with digital probes (YLC website) to encourage reflection (captured through think-out-loud); Interview to reflect on the engagement activities together with the participant. After the introduction, the participant was asked to interact with the website and try to register with YLC. Users were given approximately five minutes to register. Participants who had successfully registered with the website were asked to interact further with the mock-up to accomplish a set of tasks. The aim of these tasks was to explore topics of confidence with participants by using the privacy awareness probes.

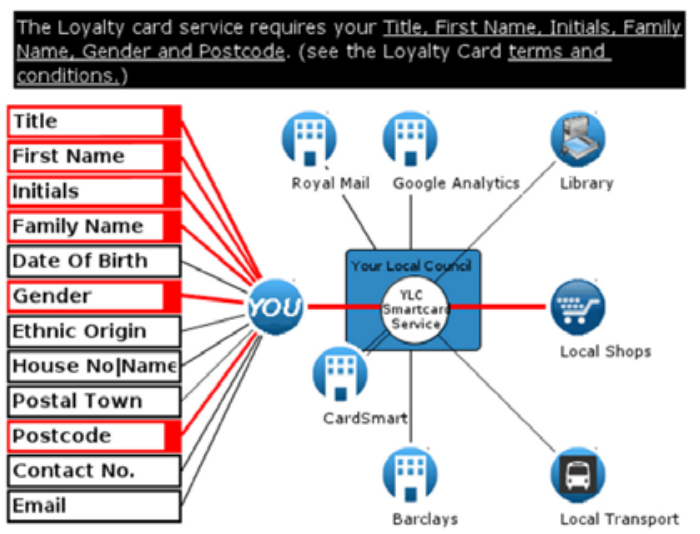

Fig. 2. ST Map

\section{Participants.}

100 (65 female and 35 male) participants recruited from 8 UK Online Centres (an organization focusing on IT training and supporting the digital inclusion of the UK public) based in London, Guildford, Bracknell, Bradford, Sheffield, and Sunderland (cities in UK). All participants (Internet users at the centre aged between 16-65 years old) 
were recruited by the Centre Manger and offered a shopping voucher as a reward for their contribution to the research. We were interested in the broad spectrum of Internet experience. $85 \%$ of the participants had more than a year's Internet experience. In addition to experienced Internet users with more than five years, we recruited 'non-users' as well. In HCI non-users are regarded as potential users, which refer to people who might in the future engage with the system but are currently inactive users [5]. Not only are "non-users" interesting in the sense that they might potentially be on their way to becoming users but also some of the participants in the initial study indicated that "nonusers”, for example grandmothers, played a role in influencing on-line behaviours of family members. As a result they are included in this study and their views on confidence and personal information disclosure elicited.

\section{Results.}

All participants engaged with the technology probes whilst completing the registration tasks. In particular, all participants engaged with the ST map. Almost 93 users felt the registration process was easy to do. However 43 users indicated they needed help in order to complete the task. A comparison between users' general opinion about websites and their specific opinion about the YLC prototype was made with Wilcoxon-pairs signed-ranks analysis $(\mathrm{p}<0.05)$ : When users interact with the prototype do they feel less concerned about their privacy compared to their general experiences with websites on the Internet? The participants confirm that this is the case. Users feel significantly less concerned when asked for information but also did not really feel that they had to think twice before submitting their information. Although the prototype gave the user no choice other than to follow the steps and deliver the required information (in comparison to opt-out/opt-in) the majority of participants $(n=68)$ perceived that the service provider gave them enough freedom to make decisions about how their information was collected, used and shared. 52 participants were of the opinion that the YLC discloses how information is collected, processed and used. Only 6 users did not agree. In other words, the majority of users perceive that the provider is making them aware of how their personal information is handled. There were also significantly less concern about how the YLC prototype takes care of the safety of their personal information. 48 users disagree that YLC does not care about the safety of their personal information. 73 
users think that a website should contain security marks indicating that the website is secure. Although YLC did not contain any security marks only 30 users disagreed that YLC was a secure website. The majority of users $(n=76)$ agrees that YLC uses information after giving consent. YLC makes people feel assured that their information is not used for different reasons. Most users $(\mathrm{n}=66)$ agreed that this is the case and appreciate the fact that the prototype was transparent concerning the way information was used. Finally, one of the important questions that supports our research questions is whether users are made aware by the prototype about how their personal information will be used. The highly significant outcome indicates that YLC is performing well (only 13 users disagreed) with regards to making people aware of how their information is used compare to other website in general.

Less-experienced users had difficulties to proceed to 'Registration Form' as they had lack of confidence in various forms. These have been categorised in: Institutional confidence; Technological confidence; and Human relationship confidence.

\section{Institutional confidence.}

The reactions to the probes demonstrated that an important method of engendering confidence is service providers' openness. For instance, Sally an experienced Internet user (age of 30 - 40) stated service provider's "openness" and "honestly" gained her confidence. Richard (age 20 - 30), was “amazed” by the way information was presented (ST map) and "impressed" by the service provider's disclosure on the process. 92 participants said they trust the service provider as it is an e-government service and they are confident the local council will keep their information confidential.

\section{Technological confidence.}

72 participants had self-confidence in being able to use the technology and also confidence that the service provider has the technology in place to protect personal information. It has long been established that confidence in technology is related to a willingness to disclose personal data. However, this study showed that there are several distinct aspects to the question of technology confidence. It is both a question of feeling confident in being able to use the technology presented to you as part of the service and also a confidence in the technology to keep any disclosed information secure. Even 
when a participant trusted the service provider's brand and had experience of that brand, they were likely to still look for signs of technological security controls. Designing for confidence requires the interface used for Information Presentation to be clear and easy to understand. Interfaces also need to be designed to support users under time pressure. Service users need to have confidence in using the technology before feeling comfortable about disclosing personal information. Julie (age 40 - 50) as a non-user was reluctant to register. She had no confidence in using the probes. Rose (age $40-49$ ) a novice Internet user preferred to continue using services off-line. Chris (age 50+) another novice user, felt very "frustrated" as he expected to see a registration form instead of the ST map. It is not only important for the service design to engender confidence in the use of the technology but also to engender confidence in the technological capability for information protection. For example, Antony (age 50+) with less than 1 year experience was looking for security signs on the website. Confidence building is not linear and media reports and publicised breaches that affect users' confidence. Richard is a young-adult who considered himself as an experienced Internet user. In the past Richard has ignored on-line privacy issues and would reveal his personal information whenever he needed a service. He stated: "I registered with the play station network which had my details and some hackers got in and stole seventy seven million people's details and so that's probably made me a little bit more cautious to the companies and what various companies do to secure data". Although he has confidence in the service provider, he has lost his confidence in their ability to protect his personal information. This demonstrates that service providers are evaluated on their technical ability to manage personal information. The design of a service needs to reflect and articulate the safety and security mechanisms in place. However, the technical evaluation can be part of the social relationship recommender network that service users often operate within. Reliance on family members to evaluate unknown service providers, particularly for female service users, was also a pattern that could be seen in the results. Pam (50+ years old) an experienced user checks the security mechanisms used on the website and this consists of checking the URL address of the website to see if it is HTTP or HTTPS (HTTP Secure). The most prominent service design features to engender a service user's own confidence could be the visibility of system status and the aesthetic qualities of the interface. 


\section{Human relationship confidence.}

Throughout the VOME studies it has been a fairly frequent comment that people seek or use personal recommendations. 70 participants said Feedback from previous users is very important element for them to make their decision about using the service. Some participant such as Carla (age 40 - 50) an experienced user were in favour of a chat system and said "immediate connection" is an important factor. Others felt having a chat system "for privacy may be a bit excessive" (Stephone, 30-39 years old, experienced user). The evident drawback which participants were mostly concerned about was incapability of the chat system to run through a voice chat as well as text. Jaali (2029 years old, experienced user) said: “....you don't know who you're talking to and that bit I didn't like... it could be weird to [chat] with somebody I didn't know, especially about something like privacy issues. It's like, I'm talking about privacy issues, but I don't know who I'm talking to." Therefore, the principle of a real-world implementation of face to face communication is important for the effectiveness of service user to service provider communication. In the case of the probe design, the anonymity of the communication and, in some cases, the lack of privacy resulted in little increase to feelings of confidence. The rule of confirmation is also an important principle if the method of communication is accepted.

\subsection{The second prototype}

The prototype (Figure 3) embedded ST map with the registration page in which a user could choose to reveal less, but losing out on some opportunities. In order to be consistent with the previous study the same research methodology used. Users' behaviours and their interaction with ST map as well as their perceptions of on-line privacy probes were recorded to test and evaluate our research hypotheses. 102 participants (aged between 18- 60 years old) were recruited through UK Online Centre of which 64 were experienced Internet users with more than 5 years. Participants were equally clustered in two groups: Group One (G1) to interact first with the second version of the prototype - YLC with privacy probes and ST map (privacy features (PF)) and then use YLC without PF. Group Two (G2) started with YLC without PF. The null hypothesis was: The order of which website they use does not have effect on their responses. 


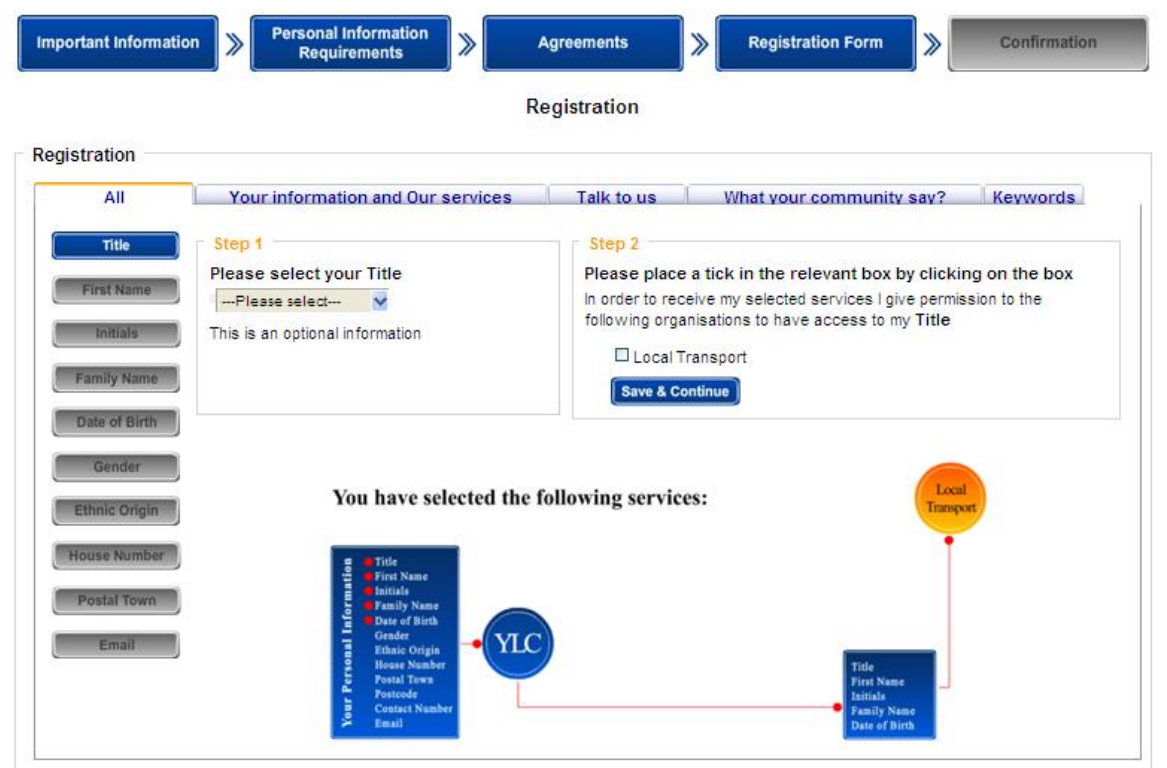

Fig. 3. Registration form with ST Map

Data collected were analysed with SPSS and T-test were applied. The result obtained from T-Test $(\mathrm{p}>0.05)$ indicates it is likely that the null hypothesis is true and the order of which participants use websites does not have effect on their responses. Therefore, our result reported here considered data gathered from G1 and G2. The result obtained from the interview, observation and questionnaire showed the presentation of privacy information (ST map) had huge impact on users' privacy awareness. 72 participants stated they are now more aware of privacy risks on-line and their attitude towards disclosure will be vigilant. 81 participants said they prefer to use an on-line registration form with ST map. More advanced Internet users said it is "time consuming" and ST map should not be embedded as part of the registration process but as a reference available for users with privacy concern. It was interesting to see 40 participants in G2 were reluctant to register with the YLC registration form without PF. 28 users from this group changed their mind and were more confident to use the service when they were exposed to the graphical information presentation in the YLC registration form with PF. All participants were satisfied with the way YLC-with PF deals with personal information. 69 participants agreed that YLC-with PF is concern about the safety and security of their user. However, 90 participants of YLC-without PF thought otherwise. 
75 participants were concerned with YLC-without PF to ask them about personal information and only 12 participants were concerned with YLC-with PF. Finally, 81 users agreed YLC-with PF gives them enough freedom to make decisions about how their information is collected, used and shared. This number was much lower for YLCwithout PF $(n=48)$.

Our research to discover more from the final user study is still on-going. Our future publication will report our further findings. Currently, we have learned transparency in privacy information and better presentation increases users' confidence in service providers' ability to protect their privacy. This increases users' privacy awareness which results in more effective engagements with on-line services.

\section{Conclusion}

How far it is economically interesting for a service provider to support confidence by design will depend on the service and the type of relationship they wish to have with their service users. Clearly, a lack of confidence has data quality and service support implications. This has cost implications for service providers needing to build close relationships with service users in order to deliver an effective service. We tested this by introducing an interactive visual map where the service provider was able to reveal its relationships with other parties and what personal information will flow to those organisations. The result obtained from our user study demonstrated that by interacting with ST map, users were encouraged to explore and gather information. Users were also more aware about what to expect and what the consequences are regarding privacy when they register for the service. Users were in favour of the map and felt more confidence. The interaction with the map also raised some interesting discussion which helped us to see other dimensions in User Confidence. This paper presented this result and discuss three distinct types of confidence: Institutional; Technological; and Relationship. The last user study focused on user confidence and user privacy awareness. Users were asked to interact with a more enhanced graphical information presentation of YLC website. The result indicated information presentation has a considerable impact on users' confidence in using on-line services. Therefore in order to help users to 
have that 'positive experience' it is important to increase user's privacy awareness though better design and transparent information.

\section{Acknowledgements}

We are grateful to all participants who took part in this study. This work as supported by the TSB; EPSRC and ESRC [grant number EP/G00255/X].

\section{References}

1. COLES-KEMP, L. AND KANI-ZABIHI, E. 2010. On-line privacy and consent: A dialogue not a monologue. In 21-23 September, ACM PRESS, Ed. ACM Press, page 1-15.

2. COLES-KEMP, L. AND KANI-ZABIHI, E. 2011. Practice Makes Perfect: Motivating confident on-line privacy protection practices. In IEEE International Conference on Privacy, Security, Risk and Trust and IEEE Internationa Conference on Social Computing, 9 - 11 October, Anonymous IEEE, , 866-871.

3. DOURISH, P., GRINTER, R.E., DELGADO DE LA FLOR, J. AND JOSEPH, M. 2004. Security in the wild: user strategies for managing security as an everyday, practical problem. Personal and Ubiquitous Computing 8, 391-401.

4. KANI-ZABIHI, E. AND COLES-KEMP, L. 2010. Service users' requirements for tools to support effective on-line privacy and consent practices. In The 15th Nordic Conference in Secure IT Systems (NordSec' 10), 24-30 October, LNCS, Ed. ACM Press, p.106-120.

5. KANI-ZABIHI, E. AND HELMHOUT, M. 2011. Increasing Service Users' Privacy Awareness by Introducing On-line Interactive Privacy Features. In Pre-Proceedings of Nordsec 2011 16th Nordic Conference on Secure IT-Systems, Talinn, Estonia, October 26-28, 2011, Anonymous, p.287-306.

6. KARAHASANOVIC, A., BRANDTZÆG, P.B., VANATTENHOVEN, J., LIEVENS, B., NIELSEN, K.T. AND PIERSON, J. 2009. Ensuring Trust, Privacy, and Etiquette in Web 2.0 Applications, p. 42-49.

7. KURIYAN, R., KITNER, K. AND WATKINS, J. 2010. ICTs, development and trust: an overview. Information Technology \& People 23, p. 216-221.

8. LUHMANN, N. 2000. Familiarity, confidence, trust: Problems and alternatives. Trust: Making and Breaking Cooperative Relations, electronic edition, Department of Sociology, University of Oxford, p.94-107.

9. OFCOM MEDIA LITERACY MATTERS. 2010. Online trust and privacy: People's attitude and behaviour.

10. SMITH, M.L. 2010. Building institutional trust through e-government trustworthiness cues. Information Technology \& People 23, 222-246.

11. U S FEDERAL TRADE COMMISSION. 2000. Privacy online: Fair information practices in the electronic marketplace: A report to Congress. Washington, DC: FTC.Retrieved June 6, 2000. 